\title{
BINARITY AND ABUNDANCE ANOMALIES IN STARS
}

\author{
A. V. TUTUKOV \\ The Astronomical Council of \\ the Academy of Sciences of the USSR \\ Pyatnitskaya str. 48, Moscow \\ 109017, U.S.S.R.
}

\begin{abstract}
About one half of all stars are close binaries whose components can efficiently interact during their evolution. The interaction of the components significantly influences the evolution and changes the chemical composition of their atmospheres. This review is devoted to the discussion of the binarity role in the creation of stars with an anomalous chemical composition of their envelopes: WR, OBN, OBC, $\mathrm{RCrB}, \mathrm{Ba}$, sdBO, Am, Fm, Ap, $\mathrm{Bp}$, helium stars, $\mathrm{CO}$ stars, $\mathrm{Na}$ giants.
\end{abstract}

\section{INTRODUCTION}

The modern theory of the chemical evolution of stars solves two main problems. The first problem is to explain the evident uniformity of the chemical composition of the most part of the stars observed what is close to the solar composition. The second problem is to understand the reasons for sometimes significant anomalies of the chemical composition of about one percent of stars. The first problem has been solved in the following way. All hydrogen and almost all helium have been formed by Big Bang. The rest of the elements are produced by massive stars and are returning into interstellar medium by asymptotic giant branch stars and supernovae. The interstellar gas is well mixed which provides for uniformity of stellar chemistry.

The spectral analysis of CP stars often displays the presence of close physical satellites. Therefore it is of interest to analyse specially the influence of binarity on chemistry of components. First of all we have no reasons to think that stars in binaries have another sequence of the main nuclear reactions of the transmutation of initial hydrogen and helium into heavier elements. This sequence really consists of the ground of stellar evolutionary process, despite the presence or the absence of a companion. It is evident that all thermonuclear reactions need very high temperatures and densities which can be attained only in deep interiors of stars. To get the chemically evolved matter on the stellar surface it is necessary to mix the unevolved envelope matter with the matter from the deep interiors of stars, to lose the external envelope with unprocessed mater or get processed matter from a close and a more evolved component. As a variant of the last possibility one can discuss the accretion of interstellar gas. One more efficient possibility to change chemical compo- 
sition of stellar atmospheres consists in the differential diffusion of heavy elements. These possibilities practically exhaust the list of the so far discussed mechanisms of the creation of chemically anomalous stellar atmospheres.

The binarity of stars is a very well-spread phenomenon in the stellar world, about half of all the stars are close binaries and the rest of them are wide ones (Abt, 1983; Massevitch and Tutukov, 1988). It is clear now that the presence of a companion can influence significantly practically all the known ways of the surface stellar chemistry changing. In the second part of this review we shall discuss in more detail these possibilities and in the third part we shall describe the main types of chemically peculiar stars (CPS).

\section{THE CHANGE OF CHEMICAL COMPOSITION OF STELLAR ENVELOPES}

\subsection{Mixing}

Convection is the most efficient mechanism providing a mixing time scale $\tau_{c} \approx(l / v)(R / l)^{2}$, where $l$ and $v$ are mixing length and turbulent velocity of convective elements, $R$ is the size of the convective zone. For a solar convective envelope, for example, it consists of several years only.

Semiconvection is a special form (turbulized pulsations) of the turbulent motion of gas inside stars when the radiative gradient of temperature only slightly $\left(\Delta \nabla \approx 10^{-4}\right)$ exceeds the adiabatic one (Dudorov and Tutukov, 1972). Semiconvection mixes the matter on the border between convective and radiative zones in the nuclear timescale of a stars. Semiconvection is important for massive $\left(M \geq 13 M_{\odot}\right)$ stars on the core hydrogen burning stage ad for stars with masses $1-7 M_{\odot}$ on the core helium burning stage (Paczynski, 1970).

The matter of a rotating star has to be slowly mixed by the meridional circulation (Sweet 1950). The timescale of this process according to Fricke and Kippenhahn (1972) is: $\tau_{c}=\tau_{K H} \alpha_{\omega}^{-1}\left(\alpha_{\omega}=\omega^{2}(2 \pi G \bar{\rho})^{-1}\right.$, where $\tau_{K H}$ is the thermal time, $\omega$ is the angular velocity and $\bar{\rho}$ is the average density of the star).

Circulation remains very important for the explanation of chemistry of Am-Fm stars where its influence competes with diffusion in the outer atmosphere. The presence of a close component leads to synchronization of axial and orbital rotation of a star. That decreases circulation flux velocities and prevents the dredge up of the matter processed in circumnuclear regions in the stellar atmosphere and makes diffusion efficient.

The mixing of radiative envelopes of quickly rotating main sequence stars with the mass above $2 M_{\odot}$ by circulation can increase $N / C$ ratio in their atmospheres (Paczynski, 1973), owing to dredge up products of incomplete hydrogen burning in circumnuclear regions. A slow growth of $N / C$ with evolution (Lubimkov 1990) can be its manifestation.

During the mass exchange between the binary components in the accretor envelope arises the shell, practically an equatorial belt with a large gradient of tangential velocities. Such shells are unstable to turbulent mixing if some dimensionless number $R i=-g *$ $d \ln \rho / d R *(d R / d v)^{2} \leq 0.25$, where $g$ is gravity, $R$ is the distance in the direction which is ortogonal to the flux with velocity $v$. If we take $d \ln \rho / d R=H_{p}^{-1}, d v / d R=v_{K}\left(\alpha H_{p}\right)^{-1}$, then $R i=\left(\alpha v_{s} / v_{K}\right)^{2}$, where $v_{K}$ is Keplerian velocity, $v_{s}$ is the local sound velocity. It is evident that for a slowly rotating star the Richardson number is low, therefore the turbulent mixing is possible. That mixing is specially important in envelopes of accreting degenerate 
dwarfs where it provides for mixing dwarf carbon and oxygen with accreted hydrogen and helium. The termonuclear runaway takes this matter away from degenerate dwarf what explains a great excess of $C N O$ elements in nova envelopes.It is of interest as well that the mass of the dwarf in the course of mass exchange does not increase with time, but even decreases.

One more mechanism of mixing can operate in components of close binaries if they are rotating even slightly non-synchroniously with the orbital rotation (Dolginov, 1974).Tidal fluxes arising inside the components are not closed in this case, therefore the matter is slowly mixing in quasi-diffusional mode. This mechanism was originally used for the amplification of the magnetic field inside binary components. Leushin (1988) has found that the nitrogen abundance in binary components is about six times as high, the carbon abundance is two times as low, and the helium abundance is about fifty percent more than in single stars. It could be interpreted now as a result of some specific for binary components mixing, for example, of the tidal nature. But there has not been so far any final conclusion about the role of tidal mixing.

Sir A. Eddington in his key monography concluded: "... diffusion ... in dwarf stars has been sufficient to effect some stratification - especially of the heaviest elements...". This idea enriched by the modern understanding of radiative pressure role remains as the real ground for the explanation of the chemistry of Am-Fm stars as well as the helium deficit in sdB and helium weak stars, horizontal branch stars possibly together with the chemistry of of envelopes of cooling degenerate dwarfs (Michaud, 1970). The numerical study of diffusion is a very complicated problem because of the significant number of different factors which influence the final quantitive result: the gravity, the stage of ionization, non-black body radiation, magnetic field, the mass loss and accretion of the matter by the stars.

Typical diffusion velocities in stellar atmospheres are very low: $v_{d} \approx 10^{-5} \mathrm{~cm} / \mathrm{s}$. Only atmospheres of slow rotating A-late B-type stars are quiet enough to make diffusion efficient. Circulation velocity in their envelopes $v_{c} \approx 10^{-5} V_{100}^{2} \mathrm{~cm} / \mathrm{s}$, where $V_{100}$ is the equtorial velocity in units of $100 \mathrm{~km} / \mathrm{s}$. The above two velocities became comparable for $V_{100} \approx 1$ (Michaud 1982). A detailed model of Charbonneau and Michaud (1988) supports this estimate. Indeed, only slowly rotating A-late B-type stars display chemical peculiarities in their atmospheres (Am, Ap, Hg-Mg stars, blue horizontal branch stars, see Fig. 1). Diffusion of helium down for MS stars excludes their pulsation. The rotation with $V_{100}>1$ prevents the diffusion of helium down for so these stars can pulsate. Such stars are known as $\delta$ Sct stars.

The density of a main sequence star photosphere, $\sim 10^{-8} \mathrm{~g} / \mathrm{cm}^{3}$ therefore the accretion or stellar wind with the rate above $M \geq 10^{-11} v_{d} \approx 10^{-16} M_{\odot} / y r$ can also prevent the diffusional settling of elements. Once more only late A-B type stars have so stable envelopes, they have no convection and no mass loss. Such stars concentrated to the main sequence, to the horizontal branch, to the sequence of helium sdB and sdO stars and to two sequences of degenerate dwarfs: helium and carbon-oxygen ones (see Fig. 1).

There are several more possibilities of mixing discussed in the literature. To get the main sequence widening for a massive star there has been proposed some additional mixing on the border of the convective core (e.g. Massevitch et al. 1979). Some turbulent mixing has been assumed operating inside our Sun to keep a low neutrino flux. The evolution of close binaries on the common envelope phase can also give a significant mixing of the matter. 
All these variants are not developed quantitatively yet. It is evident, of course, that the list of mixing mechanisms is not still complete. For example, FG Sge during several years only increases the abundance of s-process elements of about two orders of value, having (according to its surface temperature) a deep radiative envelope (Kipper, 1981). None of the possibilities discussed above could directly explain such an event.

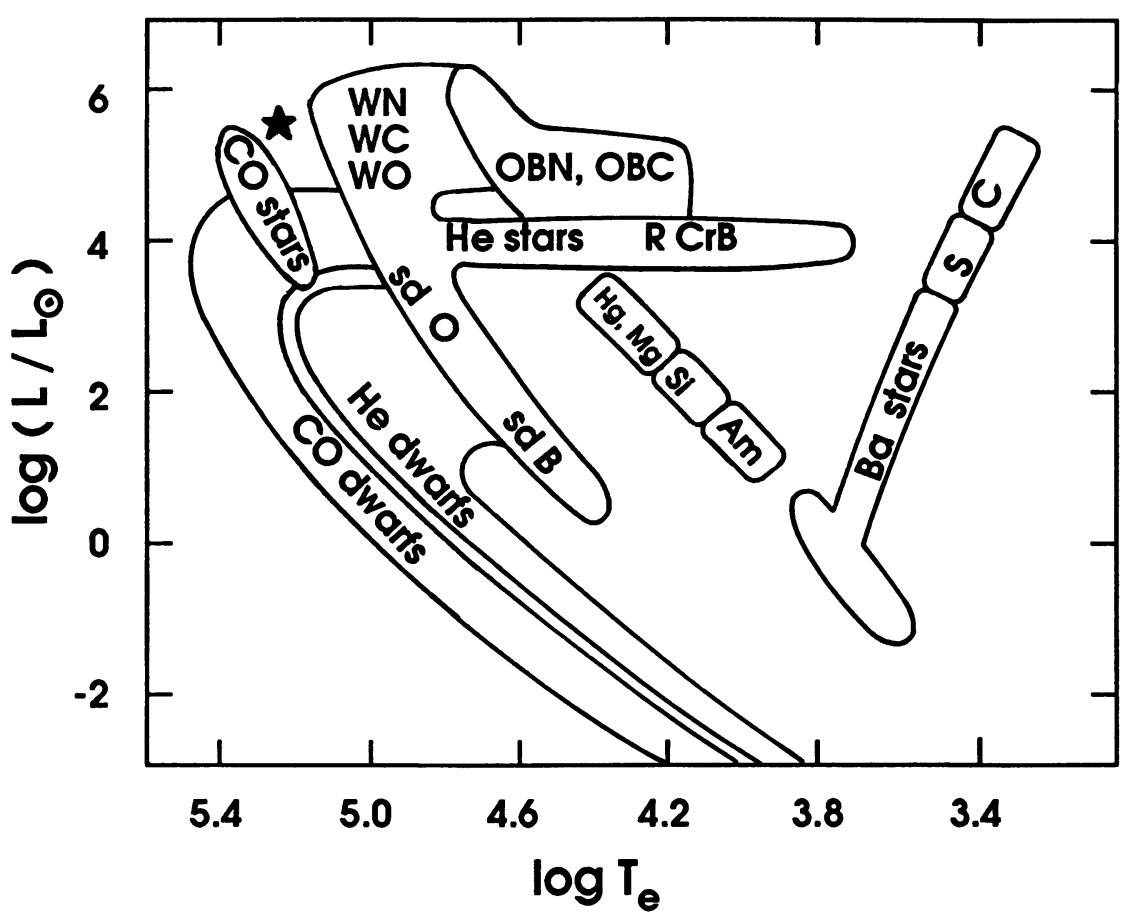

Figure 1. H-R diagram of chemically peculiar stars.

Another evidence of a possible mixing of the radiative region around the convective core of a low mass star is the chemical composition of cold giants $39 \mathrm{Cyg}$ and 9 Boo (Boyarchuk et al. 1990). The ratio ${ }^{12} C /{ }^{13} C$ for both of them is about ten, but the lithium abundances are: $[L i] \approx 2.5$ for 9 Boo and 1.15 for $39 \mathrm{Cyg}$. Whether ${ }^{7} \mathrm{Li}$ could be the result of the hydrogen burning is still unclear (Sackmann et al. 1974). Therefore contradiction to usual expectations is evident - the processed matter has lithium that is the indication of a low temperature in the past. A possible solution consists of the assumption about a slow mixing of a rather massive circumnuclear radiative shells which provides for the generation of ${ }^{13} \mathrm{C}$. The following penetration of the convective envelope inside transferred this carbon into the atmosphere, but the temperature on the bottom of the convective envelope is still not too high to burn out lithium.

Some mixing in giants can also be the result of the helium flash in the degenerate core (Deupree and Wallace, 1987). The main element dredging up from the helium core is ${ }^{12} C$. 
The differential rotation inside stars especially strong inside stars with dense nuclei could also enhance mixing, but up to now a parameter free theory of such a process is still absent.

\subsection{Accretion}

As a rule, components of close binaries have different masses. Therefore a more massive component has to be more evolved and its envelope can be enriched by matter with heavy elements which are the products of its previous chemical evolution. The mass exchange will place these products on the satellite surface. After the mass exchange the primary can finish its evolution transforming into a degenerate or a neutron star. Thus a star with an unseen and usually hardly detected companion can get an unusual for its evolution state chemistry. The mass exchange has been envolved in the discussion of the chemical composition of $\mathrm{Ba}$ stars, Algols, OBC stars.

Numerical study of the origin of massive stars displayed the possibility of a radiative separation of gas and dust (Yorke and Krugel, 1977; Tutukov and Shustov, 1979). As a result, external ten percent of mass of massive stars $\left(M \geq 10 M_{\odot}\right)$ appear almost free or deluted of dust forming elements. Some elements such as oxygen, carbon actively participate in the formation of dust, other ones like nitrogen, helium have no dust forming molecules. As a result of the accretion of the dust free matter envelopes of some massive stars can get some defficiency of carbon and oxygen simultaneously with an unchanged nitrogen abundance. But such relative abundances are usually a result of hydrogen burning in $C N O$ reactions. Thus the accretion of the dust free gas by massive stars can mimic results of hydrogen burning in massive $\mathrm{OB}$ stars. It is possible that massive stars with a relative excess of nitrogen - OBN-stars - can be explained in this way. A star with mass $M$ moving through interstellar gas accretes the latter with the rate: $M=10^{-15}\left(M / M_{\odot}\right)^{2} v_{6}^{-3} n_{H}\left(M_{\odot} / y r\right)$, where $v_{6}$ is a relative velocity in units $10^{6} \mathrm{~cm} / \mathrm{s}$ and $n_{H}$ is the number density of interstellar gas. The mass of the stellar photosphere $M_{p h}=4 \pi \rho R^{2} H_{p} \approx 8 \pi R^{2} / 3 \kappa$, where $H_{p}$ is the pressure scale, $\kappa$ is the opacity of photopshere matter and $R$ is the stellar radius. If we assume $\kappa=0.4 \mathrm{~cm}^{2} \mathrm{~g}^{-1}, R=R_{\odot}$ we shall find $M_{p h} \approx 0.5 * 10^{-10} M_{\odot}$. Therefore a star during $\tau=5 * 10^{4} v_{6}^{3}\left(M / M_{\odot}\right)^{-2} n_{H}^{-1}$ years can get a new atmosphere from interstellar gas with its chemistry. Such a possibility is very real for degenerate dwarfs since for $n_{H}^{\prime} \approx 1 \mathrm{~cm}^{-3}$ and $v_{6} \approx 3, R \approx 0.01 R_{\odot}, \tau \approx 100 \mathrm{yrs}$ only, which is a very short time comparing with Hubble time. It can explain the reason for the existence of some heavy ions in dwarf envelopes otherwise could be plunged down by the diffusion.

It is of interest to discuss the influence of such accretion on Ap-Bp stars. Because of a low angular momentum, the accreted matter moves towards the star in a rather narrow conus directed to a spot opposite to the direction of the space motion of a star. Since the star is rotating, the accreted matter will form the longitudal ring in the common sense. The width of the ring can be estimated from the solution of the corresponding problem numerically. In the limits of this ring the diffusional separation can be successfully supressed. In the presence of a strong magnetic field the selectional accretion becomes possible.

\section{CP STARS}

Gamov (1943) assumed that the excess of carbon and nitrogen in WR atmospheres was 
real and could be explained by hydrogen burning in CNO reactions inside these stars and by the mass loss with the mixing of products. Paczynski (1971) has found numerically that components of massive close binaries on the core helium burning stage have to be WolfRayet stars. The change of their chemistry from WN to WC type is entirely explained by the heavy mass loss. A part from WR stars can be formed also in the course of the evolution of single massive stars (Conti, 1976). The analysis of the galactical distribution of WR stars displays that the evolution from ON to WN stars is possible only for stars with initial masses above $40 M_{\odot}$ (Tutukov and Yungelson, 1985). Stars with initial masses $20 M_{\odot}-40 M_{\odot}$ can evolve into the WR stage only if they have close components amplifying the mass loss significantly.

The change of chemical composition during mass loss has been discussed by Tutukov and Yungelson (1980), Maeder $(1983,1984)$. A short description of the scenaria is following. An $\mathrm{O}$ star has solar abundances. When the whole initially radiative envelope is lost on the surface there appears the matter of the convective core. The ratio $N / C$ increases about hundred times and the star transforms into an $\mathrm{ON}$ star. At the moment the helium content exceeds the hydrogen content, nitrogen is still the most abundant among $C N O$ elements. This phase corresponds to WN star. Later the matter of the helium burning convective core appears on the stellar surface. Nitrogen here is almost absent, there appears an excess of carbon, therefore WC stage begins. The opening of more deep shells of the helium convective core increases the oxygen abundance and decreases the helium content. WC star transfroms itself into WO star (Rustamov and Cherepashchuk, 1988). Such is the sequence of WR stars evolution of a member of a close binary or single massive stars. Observational estimates of chemistry of WR stars supports the theoretical picture in frames of the existing uncertainties (Willis, Wilson, 1979; Nugis, 1982).

WO stars are usually in a few tens of thousand years before the explosion as supernova of probably of $\mathrm{Ib}$ type. Some of them now have no helium in their envelopes as possibly WR102 (Dopita et al. 1990). The position of this star on the HR diagram (see Fig. 1) supports such an interpretation. The absence of hydrogen and helium in the envelope assumes the absence of them in interiors too. That means that about one thousand years separate WR102 from a supernova explosion, probably type Ia, since it has neither hydrogen nor possibly helium in its envelope. So WR102 is one of the few next supernova! Thus the analysis of the chemical composition is a most sensitive indication of such presupernovae. Since the lifetime of $\mathrm{CO}$ star consists of only about one percent of the core helium burning duration, in our Galaxy there have to be about 10 of such stars.

On the analysis bases on low-noise spectra of 4 OBN stars, Schonberner et al. (1988) indicates a $N / C$ ratio higher by a factor of 100-500 than in the Sun, while $N / O$ ratio is only a factor 10-20 above solar. Simultaneously their helium content is increased by a factor 2 or 3 with respect to the solar value. Such chemistry is attainable in two ways: by means of circulational mixing or by an intense mass loss. A special study displayed that the most part of OBN stars are members of close binaries (Walborn, 1976) which possibly increases the efficiency of both mechanisms and make probable the mass exchange between components. OBC stars, as a rule, have no evidences of the presence of a close component (Walborn, 1976). Carbon excess could be the result of the previous mass exchange between components, when a more massive component was by a WC star (Tutukov, 1986). A part of the matter enriched by carbon and lost by the WC star can be intercepted by a close 
massive component. This idea would explain the $\mathrm{OBC}$ star chemistry and the absence of the close satellite observed, because the WC star after the SN explosion is now as a neutron star of a low mass and it almost does not influence the radial velocity of a massive OB star.

It is possible as well that the presence of a close massive component helps to mix stellar interiors by tidal mixing and to enrich its atmosphere with products of the hydrogen burning (Leushin 1988). That makes OBN stars. The absence of a close massive component excludes a deep mixing of the star and carbon remains, as usual, the most abundant element among CNO light elements. Thus, such stars will be the OBC stars.

$\mathrm{SdO}$ and sdB stars prolong to lower luminosities the sequence of helium non-degenerate stars on the HR diagram (Fig.1). The sdO stars have a genetic deficit of hydrogen in their atmospheres, but sdB stars have the excess of hydrogen explainable probably by diffusion of helium down during a relatively long life of the latter (Wesemael et al. 1982). sdOB stars are most populated among blue stars (Green et al., 1986). There are two main possibilities to explain the origin of those stars. One part of them are helium remnants of close binary components with initial masses $3-15 M_{\odot}$ (Tutukov, Yungelson, 1985). But the most part of sdOB stars is probably products of merging of double degenerate helium dwarfs due to the radiation of gravitational waves (Iben and Tutukov, 1985; Tutukov, 1986). These stars are relatively dim, very many of their parameters are still hardly known.

It is unclear yet whether they can pulsate. Only three stars that are pulsating with periods $0.25-1$ hour and amplitudes 0.04 are known. On the H-R diagram they are close to the sequence of helium non-degenerate stars (Bartolini et al. 1982). But now it is unknown for certain yet whether they really belong to the $\mathrm{sdBO}$ sequence or whether they are helium degenerate dwarfs contracting through this region of $H-R$ diagram to their own sequence. Additional study of these stars is necessary.

$\mathrm{R}$ CrB stars are most remarkable for recurrent deep occultations by dust clouds happening from time to time. Hydrogen is practically absent in their envelopes. They are very bright (Fig.1). R CrB stars consist of the carbon-oxygen degenerate core and extended helium envelopes (Iben and Tutukov, 1989). The total number of such stars in our Galaxy is of the order of several hundreds (Iben and Tutukov, 1985). There are two versions of their origin scenaria. Some of them could be the result of the evolution of helium remnants with masses $0.85 M_{\odot}-2.5 M_{\odot}$ of binaries components with initial masses $6 M_{\odot}-10 M_{\odot}$. But like in the case of sdBO subdwarfs most of $\mathrm{R} \mathrm{CrB}$ stars are probably products of merging of helium degenerate dwarfs with $\mathrm{CO}$ dwarfs under the influence of the gravitational wave radiation.

The ten times excess of carbon observed in the envelopes of $\mathrm{R} \mathrm{CrB}$ stars (Cotrell and Lambert, 1982) is either the result of mixing of helium with carbon-oxygen core matter during merging or the result of the thermal helium flash on the bottom of the helium envelope accreted by the CO dwarfs (Tutukov and Khokhlov, 1990).

Thus the origin of chemical peculiarities of these stars is probably entirely predetermined by the duplicity of $\mathrm{R} \mathrm{CrB}$ stars or their precedors. There are two main variants of the final fate of $\mathrm{R} \mathrm{CrB}$ stars. These stars have semi-regular mass loss. If the helium envelope is exhausted before the core mass gets the Chandrasekhar limit, the remnant will be the carbon-oxygen dwarf. If the core mass attains the critical value, the supernova will happen. The type of the supernova is not yet clear. It will not be SNII, because of the absence of hydrogen, but it is not SNI either, because of the inevitable presence of helium 
in their spectra.

A rich diversity of chemical anomalies is displayed by Ap-Bp stars belonging to the main sequence (Fig. 1). They are divided into two main subgroups: Hg-Mn and Si stars. Silicium stars, as a rule, have a strong magnetic field, Mg-Mn stars have no strong surface fields. The low limit of the magnetic stars masses is close to $2 M_{\odot}$, which is, probably, the evidence of the relic nature of their fields (Dudorov and Tutukov, 1990), since only such stars have never had a strong convection in their envelopes. Maximal mass of $\mathrm{Hg}$ - Mn stars in binaries is $5 M_{\odot}$. The duplicity rate of magnetic stars is about two-three times lower than that of usual A stars (Kraicheva et al. 1990). The duplicity of Hg-Mn stars is normal. It is probable that the strong field complicates the formation of close binaries. The low rotational velocities of magnetic stars are the result of the magnetic breaking.

All anomalies of chemical composition of Ap-Bp stars are probably concentrated in their photospheres (Ryabchikova, 1990). They are the result of the diffusion of heavy elements in gravity and radiation fields (Michaud, 1970). A low rotation is a necessary condition for the effective separation, no one has ever known Ap-Bp stars to be a member of a close binary with the period corresponding to $50 \mathrm{~km} / \mathrm{s}$. Metallic Am-Fm stars are a probable extension of peculiar stars of the main sequence to lower surface temperature (Fig. 1). They have a ten times excess of metals in the atmospheres. Their masses are $1.0 M_{\odot}-2.6 M_{\odot}$. Most of them are members of close binaries with orbital periods 1-100 days (Abt, 1983), but some of Am-Fm stars are members of wider binaries (Kraicheva et al. 1990) or single stars.

There is no necessity now to use the nuclear evolution or the mass exchange between components to explain the overabundance of metals. The presence of a close companion serves only as a way to make the rotation of $\mathrm{A}$ star slow by synchronization of axial and orbital rotations. But a single star or a member of a wide binary can be an Am-star as well, if its rotation velocity is below $100 \mathrm{~km} / \mathrm{s}$.

There are two types of atmospheres of cooling degenerate dwarfs: hydrogen rich DA and hydrogen free DB. Mass loss by AGB stars practically always leaves dwarfs with remnants of the hydrogen rich envelope. The wind can take off this matter and transform a DA dwarf into a DB dwarf. But a part of DB dwarfs can be products of close binary evolution. If the initial mass of a close binary component is in the range $5-10 M_{\odot}$ after the $\mathrm{CO}$ core in the helium remnant, the helium envelope will expand and will be lost by the star (Iben and Tutukov, 1985). A young CO dwarf with helium envelope will be formed in this case. The post $\mathrm{R}$ CrB CO dwarf will also have hydrogen free helium rich envelope. Thus, several percent of young degenerates will have helium envelopes, so the presence of hydrogen on their tops can be only a result of accretion.

Merging of double $\mathrm{CO}$ degenerates can lead to quasistationary carbon-burning in nonor semi-degenerate CO star, if the mass of the merger above $\sim M_{\odot}$ (Beaudet and Salpeter, 1969). The most part of such mergers will have masses in the range $1 M_{\odot}-2.8 M_{\odot}$ and will have to overlap on the $\mathrm{H}-\mathrm{R}$ diagram with the region of planetary nebulae nuclei. $\mathrm{CO}$ stars will probably have no nebulae around them. They could be detected among bright UV stars. The duration of the carbon burning phase is $\tau_{C O} \approx 1.5 * 10^{5}\left(M_{\odot} / M_{C O}\right)$ years. The contraction to $C$ burning is about ten times longer. Taking the frequency of their formation $\sim 0.01 \mathrm{yr}^{-1}$ and the lifetime $\sim 10^{5}$ years, we can expect about one thousand of such stars in our Galaxy. Mergers can be fast rotators. These mergers form on the H-R 
diagram together with products of heavy mass loss by WR stars (WO) a sequence of nonor semi-degenerate carbon-oxygen stars in the region of hot stars (Fig. 1).

The large gradient of angular velocity on the surface of the accreting dwarf leads to the shear mixing of the $\mathrm{CO}$ dwarf matter accreted from almost an unevolved donor. The $\mathrm{CO}$ abundance is increased about one hundred times, which has really been observed (Mustel and Baranova, 1965). Several abundant radioactive elements also arise in the process of hydrogen shell flash. Their decay can be detected in the $\gamma$ - rays range.

Barium stars exhibit overabundances of barium and of other s-process elements relative to $\mathrm{Fe}$ (Boyarchuk, 1974). Lambert (1984) demonstrated a correlation between carbon and s-process element abundances. Most of the known barium stars are giants (Fig. 1), but at least three main-sequence Ba-stars are known as M dwarf G77-61 (Dahn et al., 1977), the K5 dwarf $\epsilon$ Ind (Kollatschny, 1984) and the G8 dwarf $\xi$ Boo A (Boyarchuk and Eglitis, 1984). The spectral study of barium stars has displayed that all or almost all of them are close binaries with $300^{d} \leq P_{\text {orb }} \leq 2000^{d}$ (McClure et al. 1980; Jorissen and Mayor, 1988; McClure et al. 1985). All secondaries are degenerate dwarfs. An evidently attracting explanation for the barium syndrome origin is the mass exchange in the binary with the primary what was an evolved carbon star on AGB phase. The instability of the double shell source finally produces free neutrons and s-process elements in the primary envelope. The radius of $\mathrm{C}$-star is in rather narrow limits of $560-970 R_{\odot}$, because of the necessity to have a rather massive $\mathrm{CO}$ core (Iben and Tutukov, 1985). This provides us an estimation for the frequency of barium star formation $\sim 0.04$ per year. Thus, several percents of stars have to display barium syndrome which is close to the value observed. The accretor mass can be in the range $1 M_{\odot}-8 M_{\odot}$. So some "close relatives" of barium stars could possibly be hidden among Am-Fm and Ap-Bp stars in the main sequence. The additional study of this possibility is necessary to identify products of such mass exchange.

The common envelope formalism provides for the estimation of the orbital period of the system with barium components (Iben and Tutukov, 1985). The initial semimajor axis is about $2000 K_{\odot}$ and the accretor mass is close to $M_{\odot}$, then $P($ days $) \approx 6000\left(M_{\odot} / M_{d}\right)^{3}$. The system with the shortest periods had more massive primaries. In spite of the common envelope phase, the accretion has to be rather efficient, because of the accreted matter mixing with rather a massive convective envelope of the accretor and chemical anomalies are still significant.

Algols are a classical example of the mass exchange in binaries. Therefore the study of their chemistry is the most evident way to check theoretical predictions. Plavec (1985) has found that carbon abundance in V Sge and U CrB consists of only 9expected from the theory. The three times excess of nitrogen and a similar deficit of carbon have been discovered for U Cep by Partharathy et al. (1983). Thus, the change of CNO elements abundances is a reliable indicator of the mass exchange in algols.

The exhaustive review of the subject of $L i$ in stars was written by Michaud and Charbonneau (1990). Mixing of the convective envelope can decrease the abundance of these light elements practically to zero. tars with masses below $\sim 0.3 M_{\odot}$ are completely convective, so they have to be lithium free. Stars with masses above $1.3 M_{\odot}$ (spectrum earlier than F5) have lithium abundance $10^{-9}$ which corresponds to its content in interstellar gas. These stars have a shallow convective zone protecting burning of light elements. But Boesgaard and Tripicco (1986) have found that among F1-F9 dwarfs about $20 \%$ of stars 
have lithium depletion exceeding ten times. It is possible that some of them could be the products of merging of low mass close binaries driven by the magnetic stellar wind. A second possible explanation of the low lithium content in the envelopes of these stars could be the previous mass exchange if they are close binaries and the primary lost envelope is now a degenerate dwarf.

$\mathrm{Na}$ rich giants can be the result of the first dredge up bringing up the matter enriched by products of ${ }^{22} \mathrm{Ne}(p, \gamma)^{23} \mathrm{Na}$ reaction (Ivanov, 1990).

Stars with masses below $0.3 M_{\odot}$ are completely convective. Therefore they will have all the stable products of hydrogen burning in pp-shain reactions directly on the surface. ${ }^{3} \mathrm{He}$ abundance can be $\sim 10^{-3}$ in mass (Iben, 1967).

\section{CONCLUSIONS}

The analysis of the modern theory of close binary evolution allows one to explain origin of many stars with a peculiar chemical composition. This theory also predicts the esistence of some new types of peculiar stars like carbon-oxygena stars. The study of the stellar chemistry increases the number of the parameters describing the physical state of stars, which provides for new possibilities for the exploration of their structure and evolution. The presence of a close satellite is one of the most efficient ways for the creation of peculiar chemistry. A peculiar chemical composition can be used as an efficient discriminator of the evolutionaty state of stars. It is now important to develop a high resolution spectroscopical study of the chemical composition of different stars to compile a more complete list of chemical anomalies of stars.

The author is inbedted to A. Boyarchuk, M. Boyarchuk, N. Chugaj, T. Lozinskaya and T. Ryabchikova for many useful discussions.

\section{REFERENCES}

Abt H.A.., 1983, Ann. Rev. Astron. Astrophys., 21, 343.

Bartolini G., Bonifazi A., D'Antona F., Fusi Pecci F., Ocili L., Piccioni A., Serra R., 1982, Astorphys. Sp.Sci., 83, 287.

Beaudet G., Salpeter E.E., 1969, Astrophys.J., 155, 203.

Boesgaard A.M., Tripicco M.T., 1986, Astrophys.J., 303, 724.

Boyarchuk A.A., Eglitis I., 1984, Bull. Crimean Ast.Obs., 64, 1.

Boyarchuk A.A., 1974, "Late Stages of Stellar Evolution", eds. R.J. Taylor, p.188.

Boyarchuk M.E., Pavlenko Ya.V., Schavrina A.V., 1990, Astron. Zh. (in press).

Charbonneau P., Michaud G., 1988, Astrophys.J., 327, 809.

Conti P., 1976, Mem. Soc. Roy. Sci. Liege, 6-serie, 9, 193.

Cottrell R.L., Lambert D.L., 1982, Astrophys.J., 261, 595.

Deupree R.G., Wallace R.K., 1987, Astrophys.J., 317, 724.

Dolginov A.Z., 1974, Astron.Zh., 51, 388.

Dopita M.A., Lozinskaya T.A., McGregor P.T., Rawlings S.T., 1990, Astrophys.J. (in press).

Dudorov A.E., Tutukov A.V., 1972, Nauchn.Inform., 21, 3. 
Dudorov A.E., Tutukov A.V., 1990, Astron.Zh., 67, 342.

Dahn C.C., LiebertT., Kron R.G., Spinrad H., Hintzen P.M. 1977, Astrophys.J., 216, 757.

Eddington A.S., 1926, "Internal Construction of Stars", Cambridge Univ.

Fricke F., Kippenhahn R., 1972, Ann.Rev.Astron.Astrophys., 10, 45.

Gamov G., 1943, Astrophys.J., 98, 500.

Green R.F., Schmidt M., Liebert J., 1986, Astrophys. J. Suppl. Ser., 61, 305.

Iben I.Jr., 1967, Astrophys.J., 147, 624.

Iben I.Jr., Tutukov A.V., 1985, Astrophys.J.Suppl., 58, 661.

Iben I.Jr., Tutukov A.V., 1989, Astrophys.J., 342, 430.

Ivanov V.V., 1990, this volume.

Jorissen A., Mayor M., 1988, Astron.Astrophys., 198, 187.

Kipper T.A., 1981, Pis'ma Astron. Zh., 7, 428.

Kraicheva Z.T., Popova E.I., Tutukov A.V., Yungelson L.R., 1990, Nauchn.Inform., 68 (in press).

Kollatschny W., 1984, Astron.Astrophys., 86, 308.

Lambert D.L., 1984, Cool Stars with Excess in Heavy Elements, Eds.C. Jaschek, P.C. Keenan, 211.

Leuschin V.V., 1988, Astron.Zh., 65, 988.

Lubimkov L.S., 1990, this volume.

Maeder A., 1983, Astron.Astrophys., 120, 113.

Maeder A., 1984, Stellar nucleosynthesis, Eds. C. Chiosi, A. Renzini, p.115.

Massevitch A.G., Tutukov A.V., 1988, Evolution if stars: theory and Observation", Nauka, Moskva.

Massevitch A.G., Popova E.I., Tutukov A.V., Yungelson L.R., 1979, Astrophys.Sp.Sci., 62, 451.

McClure R.D., Fletcher J.M., Nemec J.M., 1980, Astrophys.J.Lett., 238, L35.

McClure R.D., 1985, Cool Stars With Excess of Heavy Elements. eds. M. Jaschek, P.C. Keenan, 315.

Michaud G., 1970, Astrophys.J., 160, 641.

Michaud G., 1982, Astrophys.J., 258, 349.

Michaud G., 1987, IAU Coll. No. 95 "The Second Conference on Faint Blue Stars", eds. A.G. Davis Philip et al., p.249.

Mustel E.R., Baranova L.I., 1965, Astron.Zh., 42, 42.

Nugis T., 1982, "Wolf-Rayet Stars: Observation, Physics and Evolution", eds. C. de Loore, A.J. Willis, p.127.

Michaud G., Charbonneau P., 1990, Space Science Review (in press).

Paczynski B., 1971, Ann. Rev. Astron.Astroph., 9, 183.

Paczynski B., 1970, Acta Astron., 20, 195.

Paczynski B., 1973, Acta Astron., 23, 191.

Parthasarathy M., Lambert D.L., Tomkin J., 1983, M.N.R.A.S., 203, 1063.

Plavec M.J., 1985, "Interacting Binaries", NATO Meeting, Cambridge, 155.

Rustamov D.N., Cherepashchuk A.M., 1988, Astron.Zh., 65, 1028.

Ryabchikova T.A., 1990, this volume.

Sackmann I.J., Smith R.L., Despain K.H., 1974, Astrophys.J., 187, 555. 
Schonberner D., Herrero A., Becker S., Eber F., Butler K., Kudritski R.P., Simon K.P., 1988, Astron. Ap., 197, 209.

Sweet P.A., 1950, M.N.R.A.S., 110, 548.

Tassoul J.-L., 1982, Theory of Rotating Stars.

Tutukov A.V., Khokhlov A.M., 1990, Astron.Zh. (in press).

Tutukov A.V., Yungelson L.R., 1987, "Faint Blue Stars", eds. A.G. Davis Philip et al., 435.

Tutukov A.V, 1986, "Hydrogen Deficient Stars", p.483.

Tutukov A.V., 1986, Circular of Shemakha Observatory, No.74, 6 (in Russian).

Tutukov A.V., Yungelson L.R., 1980, Astron.Zh., 57, 1266.

Tutukov A.V., Shustov B.M., 1979, Nauchn.Inform., 46, 111.

Tutukov A.V., Yungelson L.R., 1985, Astron.Zh., 62, 111.

Yorke H.W., Krugel E., 1977, Astron.Astrophys., 54, 183.

Walborn N.R., 1976, Astrophys.J., 205, 419.

Wessemael F., Winget D.E., Gabot W., Van Horn H.M., Fontaine G., 1982, Astrophys.J., 254, 221.

Willis A.J., Wilson R., 1979, IAU Symp. 83, p.461. 